\title{
Finding the needle in the haystack
}

The hunt for cancer biomarkers has, since its inception, promised to reduce cancer mortality by enabling detection and subsequent intervention at earlier, more treatable stages of the disease. Proteomicsbased approaches have dominated the field, but these have failed to realize their potential, resulting in an urgent need for novel strategies. Enter Muneesh Tewari and colleagues, whose recent results demonstrate that the detection of microRNAs (miRNAs) in serum or plasma might be an effective strategy for cancer detection.

The authors first verified that endogenous miRNAs could be detected in plasma. Total RNA isolated from the plasma of healthy subjects was radiolabelled and subjected to PAGE followed by reverse transcriptase PCR and cDNA sequencing. These experiments demonstrated that the majority of small $\sim 22$ nucleotide RNA species present corresponded to known miRNAs. Moreover, levels of three endogenous miRNAs $-m i R-15 b$, $m i R-16$ and $m i R-24$ - remained constant after subsequent freezethaw cycles, which is indicative of stability, a prerequisite for any potential biomarker.

The next challenge was to demonstrate that tumour-derived miRNAs could be detected in plasma. The authors addressed this by isolating RNA from the plasma of mice with human prostate cancer xenografts that had been growing for 28 days. Taqman quantitative real-time PCR (qRT-PCR), showed the presence of two miRNA sequences, $m i R-629^{*}$ and $m i R-660$, in experimental but not control animals. As both these miRNAs lack murine homologues they were considered to be specific, tumourderived markers. Significantly, their levels correlated with tumour burden, further reinforcing the validity of this approach.

Will this approach work in humans? The authors compiled a panel of six putative miRNA biomarkers based on published miRNA expression profiles in prostate cancer and used TaqMan qRT-PCR to compare levels of these miRNAs in the serum of metastatic prostate cancer patients with those in healthy subjects. Expression of

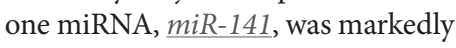
increased (46-fold) in the prostate

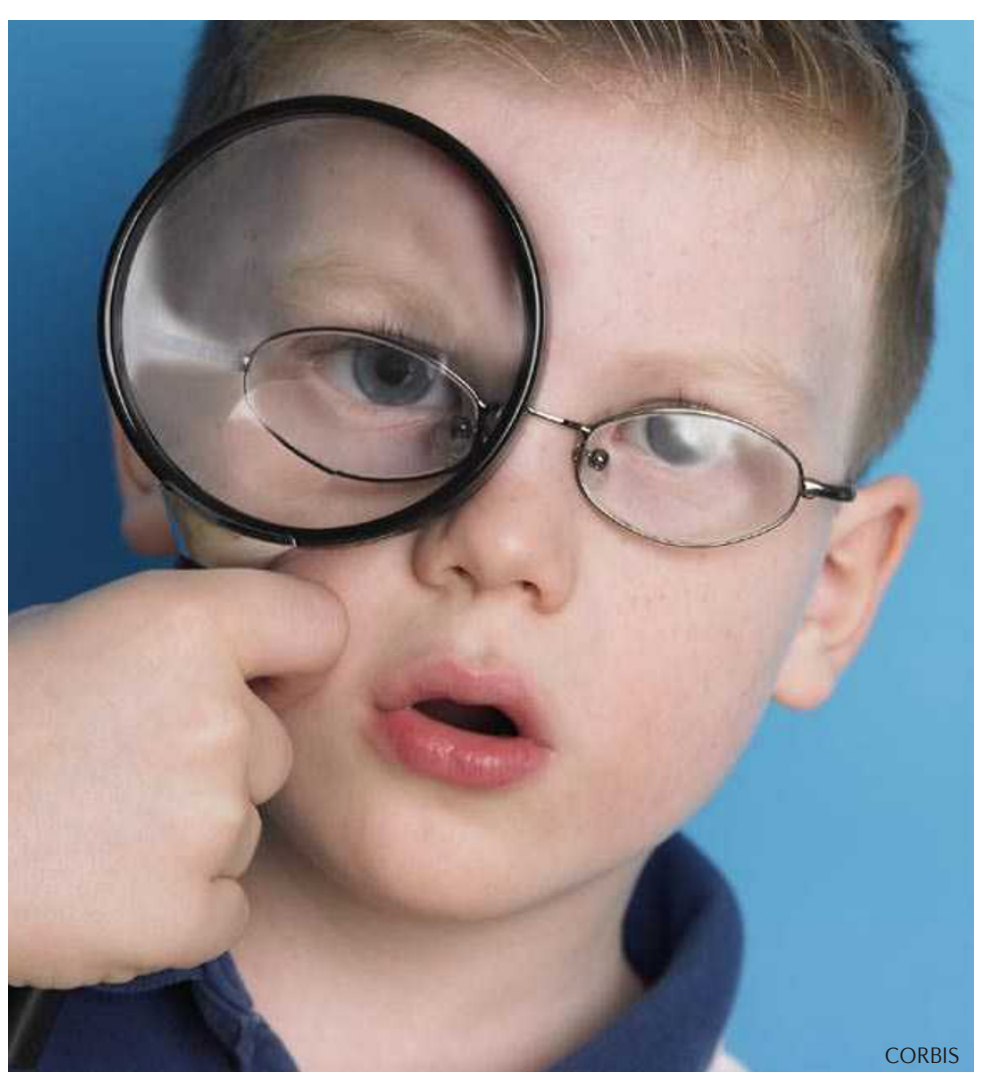

cancer group and could specifically and sensitively distinguish healthy serum from that of prostate cancer patients. Together, these data lend credence to the development of circulating miRNAs as blood-based biomarkers for cancer and suggest that miR-141 might be a promising avenue for the early detection of prostate cancer.

Safia Ali Danovi

ORIGINAL RESEARCH PAPER Mitchell, P.S. et al. Circulating microRNAs as stable bloodbased markers for cancer detection. Proc. Natl Acad. Sci. USA 105, 10513-10518 (2008) FURTHER READING Ludwig, J. A. et al. Biomarkers in cancer staging, prognosis and treatment selection. Nature Rev. Cancer 5, 845-856 (2005) 